\title{
Space-charge-limited flows in the quantum regime ${ }^{a)}$
}

\author{
L. K. Ang ${ }^{\text {b) }}$ and W. S. Koh \\ School of Electrical and Electronic Engineering, Nanyang Technological University, Singapore 639798 \\ Y. Y. Lau \\ Department of Nuclear Engineering and Radiological Sciences, University of Michigan, Ann Arbor, \\ Michigan 48109
}

T. J. T. Kwan

Applied Physics Division, Los Alamos National Laboratory, Los Alamos, New Mexico 87545

(Received 21 October 2005; accepted 16 November 2005; published online 8 May 2006)

\begin{abstract}
This paper reviews the recent developments of space-charge-limited (SCL) flow or Child-Langmuir (CL) law in the quantum regime. According to the classical CL law for planar diodes, the current density scales as 3/2's power of gap voltage and to the inverse squared power of gap spacing. When the electron de Broglie wavelength is comparable or larger than the gap spacing, the classical SCL current density is enhanced by a large factor due to electron tunneling and exchange-correlation effects, and there is a new quantum scaling for the current density, which is proportional to the $1 / 2$ 's power of gap voltage, and to the inverse fourth-power of gap spacing. It is also found that the classical concepts of the SCL flow such as bipolar flow, transit time, beam-loaded capacitance, emitted charge density, and magnetic insulation are no longer valid in quantum regime. In the quantum regime, there exists a minimum transit time of the SCL flows, in contrast to the classical solution. By including the surface properties of the emitting surface, there is a threshold voltage that is required to obtain the quantum CL law. The implications of the Fowler-Nordheim-like field emission in the presence of intense space charge over the nanometer scale is discussed. (C) 2006 American Institute of Physics. [DOI: 10.1063/1.2174834]
\end{abstract}

\section{INTRODUCTION}

Space-charge-limited (SCL) flow has been an area of active research in the development of non-neutral plasma physics, high current diodes, high power microwave sources, vacuum microelectronics, and sheath physics. The classical SCL electron flow [also known as the Child-Langmuir (CL) law $\left.{ }^{1,2}\right]$ gives the maximum current density allowed for steady-state electron beam transport across a gap with gap spacing $D$ and gap voltage $V_{g}$. According to the classical CL law for the planar diodes, the SCL current density scales according to 3/2's power of gap voltage and the inverse squared power of gap spacing. The classical CL law is essentially a one-dimensional (1D) law for nonrelativistic electron flow across a planar gap at zero emission velocity, and it is given by

$$
J_{C}(1 \mathrm{D})=\frac{4 \epsilon_{o}}{9} \sqrt{\frac{2 e}{m_{e}}} \frac{V_{g}^{3 / 2}}{D^{2}},
$$

where $e$ and $m_{e}$ are the charge and mass of the electron, respectively, and $\epsilon_{o}$ is the permittivity of free space.

Since the pioneering work of Child and Langmuir, the 1D planar CL law has been improved to include new physics in various models, such as coaxial cylindrical diodes, ${ }^{3}$ finite initial velocity, ${ }^{4}$ SCL bipolar flow, ${ }^{5}$ relativistic voltage (without self-magnetic field) ${ }^{6}$ electron tunneling, ${ }^{7}$ short-pulse, ${ }^{8}$ collisions, ${ }^{9}$ stochastic coulomb interactions, ${ }^{10}$ saturation, ${ }^{11}$

\footnotetext{
a) Paper LI2 4, Bull. Am. Phys. Soc. 50, 222 (2005).

${ }^{b)}$ Invited speaker. Electronic mail: elkang@ntu.edu.sg
}

open cavity diodes, ${ }^{12}$ and vacuum capacitance formulation. ${ }^{13}$ In particular, extensive studies have been done on extending the classical 1D CL law to two-dimensional (2D) models both numerically and analytically, ${ }^{14-19}$ and its development may be found in a recent review paper. ${ }^{20}$ More recently, the three-dimensional (3D) model has also been developed for a wide range of $V_{g}$ and $D$ (i.e., for quantum, classical, and weakly relativistic regimes). ${ }^{21}$

With the rapid progress in nanoscale fabrication, nanosize gaps between metallic electrodes have been successfully fabricated. ${ }^{22-24}$ Nanodiodes and nanotriodes, ${ }^{22,23}$ which can operate at low voltage $V_{g}<40 \mathrm{~V}$ with gap spacing on the order of $D<100 \mathrm{~nm}$, have been fabricated. Using nanoscale emitters, high current $(>1 \mu \mathrm{A}$ per tip) can be obtained with low voltage at room temperature, which corresponds to a very high local electron current density $\left(>10^{8} \mathrm{~A} / \mathrm{cm}^{2}\right)$ transport across a nanogap.

Thus, it is of interest to study such a high current electron ballistic transport in nanoscale like SCL electron flow in a nanogap with low voltage, where the quantum effects may become important. ${ }^{21,25-27}$ By including quantum effects such as the electron tunneling and many electron exchangecorrelation interaction, new quantum CL law ${ }^{25,26}$ has been developed in the operating regime where electron de Broglie wavelength is comparable to the gap spacing. Using mean field theory and local density theory, the SCL electron current density across a nanogap is calculated for a given $D$ and $V_{g}$. New quantum scalings of $V_{g}^{1 / 2}$ and $D^{-4}$ have been calculated both numerically ${ }^{25}$ and analytically. ${ }^{26}$ It is also found 
that the diode is no longer completely magnetically insulated in a crossed-field nanogap due to electron tunneling. ${ }^{27}$

This paper gives an overview of the quantum CL law, and also presents some recent extensions of the models in understanding the properties of SCL flows in the quantum regime, such as beam-loaded capacitance, transit time, and surface properties, which have not been studied before. The paper will show that these classical concepts of SCL flows are no longer valid when quantum effects become important. In particular, we found that there is a minimum electron transit time (in contrast to the classical solution) due to electron tunneling. Including the surface properties, we also found that a threshold voltage is required to overcome the work function of the emitting surface to reach the quantum $\mathrm{CL}$ law. Smooth transition between the classical and quantum regimes will be demonstrated.

The work presented herein is motivated by two distinct but related goals. First, the fundamental study of high current electron interaction in quantum regime (such as quantum $\mathrm{CL}$ law studied in this paper) offers an opportunity to exploit an entirely different physical regime in which the classical theory fails. Thus all the conventional classical concepts that were used in vacuum devices such as limiting current, beam loading, beam modulation, detuning of a cavity, frequency response, and noise may require a complete revision. The second goal focuses on developing high current and stable cathode by using novel field emission cathode, such as carbon nanotube (CNT) or other nanoscale emitters. To correctly account for the space-charge effects of the emitted current close to the nanoscale emitter, a new model is required to provide a consistent transition from FowlerNordheim (FN) emission (at low current regime) to SCL flow (at high current regime). Thus the understandings of nanophysics on electron emission close to the cathode's surface may be crucial in developing high current cathodes for various applications.

The rest of the paper is organized as follows. Section II reviews the 1D quantum model of SCL flow based on meanfield theory and local density theory. Using the quantum model, classical theories of bipolar SCL flow, transit-time, beam-loaded capacitance, magnetic insulation and transition from field emission to SCL flow are revised to include quantum effects. The paper is concluded in Sec. III.

\section{QUANTUM MODEL OF SCL FLOW}

Let us consider a 1D planar nanogap of gap spacing $D$ with a grounded cathode and an anode potential of $V_{g}$. Electrons and ions with zero initial velocity are injected normally into the gap from the cathode and anode respectively. Using mean field theory, the 1D time-independent Schrödinger equation, the Poisson equation, and charge conservation relation are solved to obtain the maximum electron current density $J$ and ion current density $J_{i}$ that can be transported under the space-charged-limited (SCL) condition. The respective 1D time-independent Schrödinger equation, and the Poisson equation are

$$
-\frac{\hbar^{2}}{2 m} \frac{d^{2} \psi}{d x^{2}}=\left(e V-V_{\mathrm{xc}} \times E_{H}\right) \psi
$$

$$
\frac{d^{2} V}{d x^{2}}=\frac{e \psi \psi^{*}}{\epsilon_{o}},
$$

where $V_{\mathrm{xc}}$ is the electron exchange-correlation potential (in terms of the Hartree energy $E_{H}$ ) that is calculated by KohnSham density functional theory (DFT) (under random phase approximation), ${ }^{28}$ and $\psi$ is the complex electron wave function.

For simplicity, we introduce several normalized parameters: $\bar{x}=x / D, \phi=V / V_{g}, \lambda=D / \lambda_{o}$ is the normalized gap spacing, $\phi_{g}=e V_{g} / E_{H}$ is the normalized gap voltage, $\psi / \sqrt{n_{o}}=q$ $\times \exp (i \theta)$ is the normalized wave function, where $q$ and $\theta=\frac{2}{3} \gamma \times \lambda \times \int_{1}^{\bar{x}} q^{-2} d \bar{x}+\theta(1)$ are, respectively, the real functions of the wave amplitude and phase, and $\gamma=J / J_{\mathrm{CL}}$ is the normalized electron current density. The normalized scales: $\lambda_{o}=\sqrt{\hbar^{2} / 2 e m_{e} V_{g}}$ is the electron De Broglie wavelength at $V_{g}, \quad n_{o}=2 \epsilon_{o} V_{g} / 3 e D^{2}$ is the electron density scale, $E_{H}=e^{2} / 4 \pi \epsilon_{o} a_{o}=27.2 \mathrm{eV}$ is the Hartree energy, and $a_{o}=4 \pi \epsilon_{o} \hbar^{2} / m e^{2}=0.0529 \mathrm{~nm}$ is the Bohr radius.

In terms of the normalized parameters, the $1 \mathrm{D}$ timeindependent Schrödinger equation, the Poisson equation and charge conservation relation can be formulated as

$$
\begin{aligned}
& q^{\prime \prime}+\lambda^{2}\left(\phi-\frac{V_{x c}}{\phi_{g}}-\frac{4}{9} \frac{\gamma^{2}}{q^{4}}\right) q=0, \\
& \phi^{\prime \prime}=\frac{2}{3} q^{2}-\frac{1}{4} \frac{\alpha}{\sqrt{1-\phi}}, \\
& \alpha=\phi^{\prime}(0)^{2}-\phi^{\prime}(1)^{2}+\frac{4}{3} \int_{0}^{1} q^{2} \phi^{\prime} d \bar{x},
\end{aligned}
$$

where the prime denotes the derivative with respect to $\bar{x}$. The parameter

$$
\alpha=\frac{16}{9} \sqrt{\frac{m_{i}}{Z m_{e}}} \frac{J_{i}}{J_{\mathrm{CL}}}
$$

is the normalized ion current density $J_{i}$, where $Z$ and $m_{i}$ are the charge and mass of the positively charged ion respectively. The boundary conditions for Eqs. (4)-(6) are $q(1)$ $=\sqrt{2 \gamma / 3}, q^{\prime}(1)=0, \phi(0)=0$, and $\phi(1)=1$. The normalized electric field at the cathode and anode are, respectively, $\phi^{\prime}(0)$ and $\phi^{\prime}(1)$, which are numerically calculated for a given $\gamma$ and $\alpha$. With the boundary conditions, we determine the SCL electron current density and ion current density through the maximum value of $\gamma$ and $\alpha$, which are defined as $\gamma_{Q}$ and $\alpha_{Q}$. For $\gamma>\gamma_{Q}$ and $\alpha>\alpha_{Q}$, solutions to Eqs. (4)-(6) no longer exist.

\section{A. Unipolar and bipolar SCL flow}

For quantum CL law (or SCL unipolar electron flow) at $\alpha=0,{ }^{25}$ Eq. (6) becomes

$$
\phi^{\prime}(1)^{2}-\phi^{\prime}(0)^{2}=\frac{4}{3} \int_{0}^{1} q^{2} \phi^{\prime} d \bar{x},
$$

which gives the relation between $\phi^{\prime}(0) \leqslant 0$ and $\phi^{\prime}(1)$ $\geqslant 4 / 3$ to calculate $\gamma_{Q}$ as a function of $\lambda$ and $\phi_{g}$. In the 

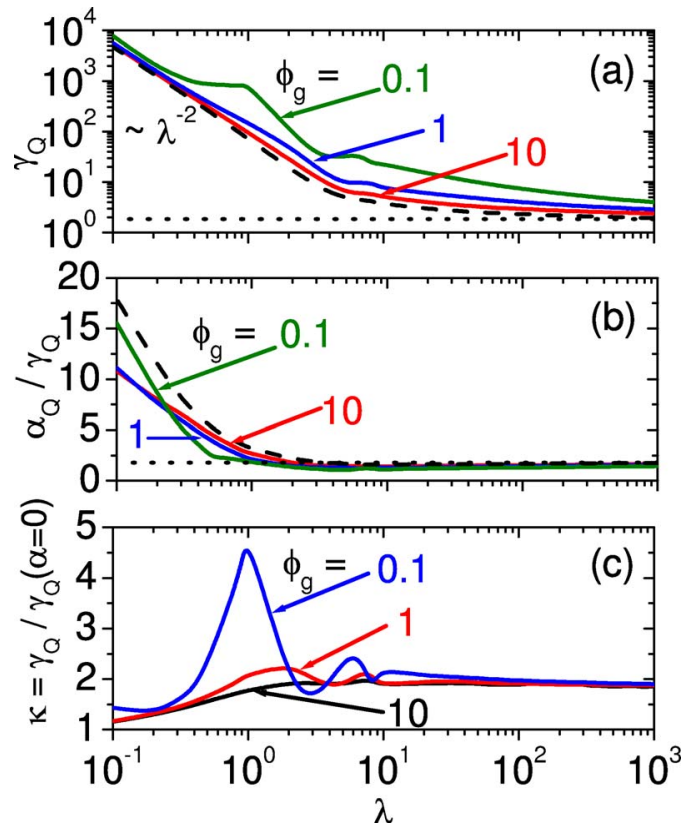

FIG. 1. (Color online) The dependence of the following parameters as a function of $\lambda$ for $\phi_{g}=0.1,1$, and 10 (solid lines) and $\phi_{g} \gg 1$ (dashed line): (a) The normalized bipolar SCL electron current density $\gamma_{Q}=J / J_{\mathrm{CL}}$, (b) the normalized ion current density (in terms of electron current density) $\alpha_{Q} / \gamma_{Q}$, and (c) the ratio of the bipolar electron current density to the unipolar electron current density, $\kappa=\gamma_{Q} / \gamma_{Q}(\alpha=0)$. The dotted line is the classical value of (a) $\gamma_{Q}=1.86$ and (b) $\alpha_{Q} / \gamma_{Q}=16 / 9$

classical limit at $\lambda \gg 1$ and $\phi_{g} \gg 1$, we have $\phi^{\prime}(0)=0$, $\phi^{\prime}(1)=4 / 3$, and $\gamma_{Q}=1$.

For SCL bipolar flow ${ }^{29}$ at finite $\alpha_{Q}$, we ignore completely the quantum effects of ions by assuming that the De Broglie wavelength of ion is much smaller than the gap spacing. Thus the SCL ion flow is limited by the zero electric field at the anode, $\phi^{\prime}(1)=0$, and Eq. (6) becomes

$$
\alpha_{Q}=\phi^{\prime}(0)^{2}+\frac{4}{3} \int_{0}^{1} q^{2} \phi^{\prime} d \bar{x},
$$

for which $\gamma_{Q}$ and $\alpha_{Q}$ are determined by varying $\phi^{\prime}(0) \leqslant 0$. In the classical regime, the solution will approach the classical limit of $\phi^{\prime}(0)=0, \gamma_{Q}=1.86$, and $\alpha_{Q} / \gamma_{Q}=16 / 9$ (or $J_{i} / J$ $=\sqrt{\left.Z m_{e} / m_{i}\right)}$.

Figures 1(a) and 1(b) illustrate the dependence of $\gamma_{Q}$ and $\alpha_{Q} / \gamma_{Q}$ as a function of $\lambda$ for $\phi_{g}=0.1$ to $\phi_{g} \gg 1$, where the classical values are also plotted for comparison (dotted lines). For a given $\phi_{g}$, both $\gamma_{Q}$ and $\alpha_{Q}$ increase significantly with decreasing values of $\lambda(<10)$ in the quantum regime. At very small $\lambda$ (deep quantum regime), the value of $\gamma_{Q}$ is approximately proportional to $\lambda^{-2}$. Thus, similar to the quantum CL law, ${ }^{25}$ the quantum bipolar electron flow also has a quantum scaling of $V_{g}^{1 / 2}$ and $D^{-4}$ as opposed to the classical scaling of $V_{g}^{3 / 2}$ and $D^{-2}$. Note this quantum scaling have also been proved by a simple derivation based on a dimensional argument of the Schrödinger equation and the Poisson equation without including the exchange-correlation term. ${ }^{26}$ From the figure, we see that the amount of the SCL ion current density (in terms of electron current density) is no longer a constant, which is $\alpha_{Q} / \gamma_{Q}=16 / 9$ as predicted by the classical theory.

From Fig. 1(c), we study the degree of charge neutralization in various $\lambda$ and $\phi_{g}$ by plotting the ratio of SCL electron current density of the bipolar flow to the unipolar flow, $\kappa=\gamma_{Q} / \gamma_{Q}(\alpha=0)$. In the quasiclassical regime $(\lambda=1000), \kappa$ approaches the classical limit of 1.86 as expected. At small $\lambda<0.1, \kappa$ decreases to about 1.15-1.5 in the deep quantum regime. In the range of $0.1<\lambda<100$, the ratio depends explicitly on the values of $\lambda$ and $\phi_{g}$, which is due the exchange-correlation effects, especially for small $\phi_{g}$. Thus, we may conclude that the space-charge effects of ions may be negligible at small $\lambda<0.1$, where the electron tunneling is more significant and the bipolar flow is nearly identical to the unipolar flow (or quantum CL law) ${ }^{25}$ with a small enhancement of 1.15-1.5 that is even smaller than the classical limit of 1.86 .

\section{B. Capacitance and transit time}

The quantum SCL flow can also be formulated using a capacitance model, where the beam-loaded capacitance of the nanogap and the transit time under SCL conditions are calculated. For simplicity, we will only focus on the SCL unipolar electron flow to calculate $\gamma_{Q}$ at $\alpha_{Q}=0$ and its related properties for the rest of the paper.

Consider that the SCL electron current density can be written in the form of $J=q_{e} / T$, where $q_{e}$ is the emitted electron charge (per area) being transported across the gap and $T$ is the transit time. From charge conservation, we have $q_{e}=q_{c}+q_{a}$ which equals to the sum of the surface charge density on the cathode and anode, respectively. By using the Gauss law, the $q_{c}$ and $q_{a}$ can be determined from the amplitude of the surface electric field on the electrodes. Using $C=d q_{e} / d V_{g}$, we may also determine the beam-loaded capacitance at the SCL condition for a given $V_{g}$ and $D$.

In terms of normalized parameters, the normalized transit time $(\tau)$ and beam-loaded capacitance $\left(C_{q}\right)$ are

$$
\begin{aligned}
& \tau=\frac{3}{4} \frac{\sigma}{\gamma_{Q}}, \\
& C_{q}=\frac{2}{3} \sigma+\frac{2}{3} \phi_{g} \frac{d \sigma}{d \phi_{g}}, \\
& \sigma=\int_{0}^{1} q^{2} d \bar{x}=\frac{3}{2}\left[\phi^{\prime}(1)-\phi^{\prime}(0)\right],
\end{aligned}
$$

where $\sigma=q / \sigma_{o}$ is the normalized emitted electron charge density (per area), $\tau=T / t_{o}$, and $C_{q}=C / C_{o}$. The normalized constants are $\sigma_{0}=e n_{o} D=2 \epsilon_{o} V_{g} / 3 D, t_{o}=2 D / \sqrt{2 e V_{g} / m}$ is the average transit time for a single classical electron, and $C_{o}=\epsilon_{o} A / D$ is the vacuum capacitance of a parallel plate. By combining Eq. (7) and (11), we may also calculate the average normalized electric field $\left\langle\phi^{\prime}\right\rangle$ by 

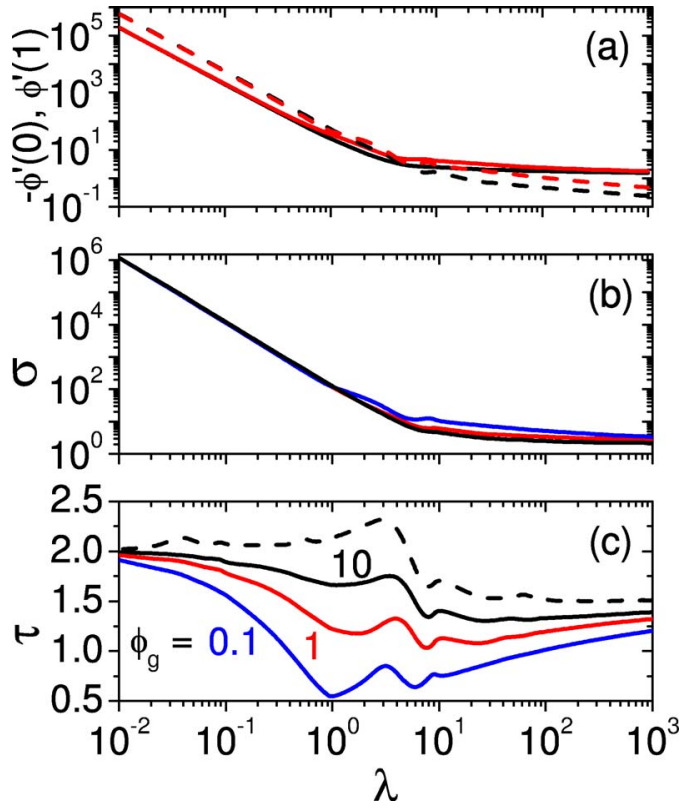

FIG. 2. (Color online) The dependence of the following parameters as a function of $\lambda$ : (a) The normalized electric potential at the cathode $\phi^{\prime}(0)$ (dashed lines) and the normalized electric potential at the anode $\phi^{\prime}(1)$ (solid lines) for $\phi_{g}=0.1$ and 1 (top to bottom), (b) the normalized free electron charge density (per unit area) for $\phi_{g}=0.1,1$, and $\gg 1$ (top to bottom), and (c) the normalized transit time $\tau$ for various $\phi_{g}=0.1,1,10$ (bottom to top), and $\gg 1$ (dashed line).

$$
\left\langle\phi^{\prime}\right\rangle=\frac{\int_{0}^{1} q^{2} \phi^{\prime} d \bar{x}}{\int_{0}^{1} q^{2} d \bar{x}}=\frac{\phi^{\prime}(1)+\phi^{\prime}(0)}{2},
$$

which simply equals to the mean value of the surface electric fields on the electrodes. For a given $\phi_{g}$ and $\lambda$, we may calculate the transit time and beam-loaded capacitance for SCL electron flow in the quantum regime. For comparison, the classical limits are $q^{2}=\frac{2}{3} \bar{x}^{-2 / 3}, \phi^{\prime}=\frac{4}{3} \bar{x}^{1 / 3}, \phi^{\prime}(0)=0 \quad \phi^{\prime}(1)$ $=4 / 3, \sigma=2, \gamma_{Q}=1,\left\langle\phi^{\prime}\right\rangle=2 / 3, \tau=3 / 2$, and $C_{q}=4 / 3$, which are independent of $V_{g}$ and $D$.

Figure 2 illustrates the dependence of $\phi^{\prime}(1),-\phi^{\prime}(0), \sigma$, $\tau$ as a function of $\lambda$ for various $\phi_{g}$. For a given $\phi_{g}$, both $\phi^{\prime}(1)$ (solid lines) and $-\phi^{\prime}(0)$ (dashed lines) increase significantly with decreasing values of $\lambda(<10)$ in the quantum regime. In Fig. 2(b), the emitted charge density of the SCL electron flow, $\sigma=\int_{0}^{1} q^{2} d \bar{x}$ is plotted, which agrees very well with the relation of $\frac{3}{2}\left[\phi^{\prime}(1)-\phi^{\prime}(0)\right]$ derived in Eq. (11). From both figures, we can see that the quantum effects become significant at $\lambda<10$. For large $\lambda>10$, we have $\phi^{\prime}(1)$ $>-\phi^{\prime}(0)$, so the average electric field $\left\langle\phi^{\prime}\right\rangle$ [see Eq. (12)] is positive, so quantum effects is less significant. However at small $\lambda<10,\left\langle\phi^{\prime}\right\rangle$ becomes negative when $\phi^{\prime}(1)<-\phi^{\prime}(0)$, which implies significant electron tunneling in the gap.

From Fig. 2(c), there is a minimum transit time (or tunneling time) at certain values of $\lambda$ for low $\phi_{g}$. Using Eqs. (9) and (11), the normalized transit time is $\tau=\frac{3}{4} \int_{0}^{1} q^{2} d \bar{x} / \gamma_{Q}(q)$, which defines the time a quantum electron takes to transit across the gap or the time required by the SCL current $\gamma_{Q}$ to
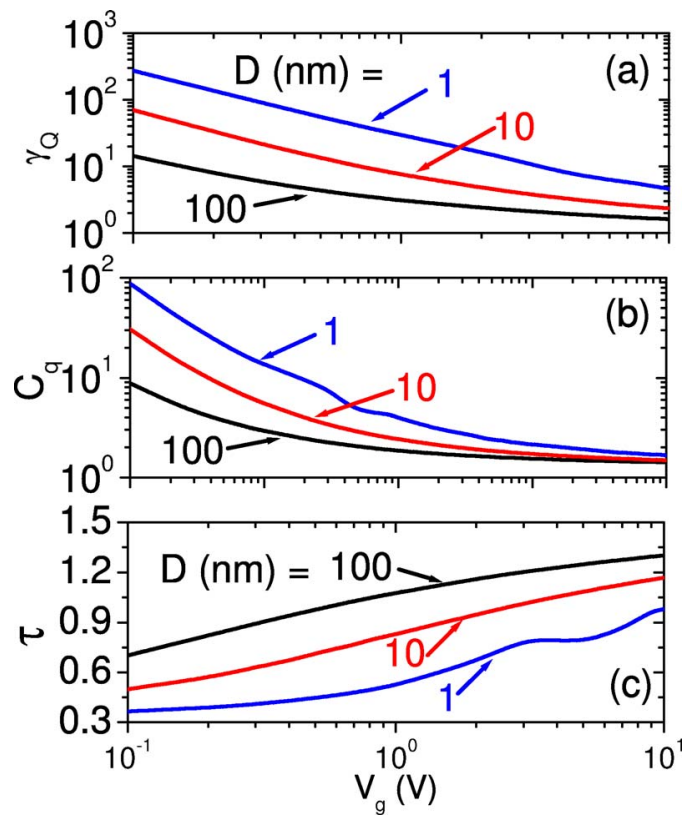

FIG. 3. (Color online) (a) The normalized unipolar SCL electron current density $\gamma_{O}=J / J_{\mathrm{CL}}$, (b) the normalized beam-loaded capacitance $C_{q}$, and (c) the normalized transit time $\tau$, as a function of $V_{g}$ for $D=1,10$, and $100 \mathrm{~nm}$.

replace all the electrons in the gap with new ones. ${ }^{30}$ It is clear that $\tau$ is definitely a positive quantity that is bounded from below. Thus, one can find some specific solutions of wave amplitude $q(\bar{x})$ for which the $\tau$ assumes its minimum value at certain intermediate values of $\lambda$ for small $\phi_{g}$ as shown in the Fig. 2(c). For $\phi_{g}=0.1$, we have $\tau \approx 0.5$ at $\lambda \approx 1$, which is smaller than the classical limit of $\tau \approx 1.5$ at $\lambda \gg 1$ and the deep quantum limit of $\tau \approx 2$ at $\lambda \ll 1$.

In Fig. 3, the calculated values of $\gamma_{Q}, C_{q}, \sigma$, and $\tau$ are plotted in the range of $V_{g}=0.1-10 \mathrm{~V}$ for $D=1,10$, and $100 \mathrm{~nm}$ to illustrate some practical parameters that can be fabricated with the current nanofabrication technology. The results show that the quantum calculations differ significantly from the classical limits of $\gamma_{Q}=1, C_{q}=4 / 3$, and $\tau=3 / 2$.

\section{Magnetic insulation}

In the classical regime, an electron emitted from cathode is prohibited from reaching the anode when a crossed magnetic field $(B)$ larger than the Hull cutoff magnetic field, $B_{H}=\sqrt{2 m V_{g} / e D^{2}}$ is applied. ${ }^{31}$ However, the diode is no longer completely magnetically insulated at $B>B_{H}$ in the quantum regime for a crossed-field nanogap due to the finite probability of electrons tunneling through the potential barrier to arrive at the anode. ${ }^{27}$

To account for the effects of the crossed magnetic field, an additional term of $-\left[\left(B / B_{H}\right) \bar{x}\right]^{2}$ is added into Eq. (4), which becomes

$$
q^{\prime \prime}+\lambda^{2}\left(\phi-\frac{V_{\mathrm{xc}}}{\phi_{g}}-\frac{4}{9} \frac{\gamma^{2}}{q^{4}}-\frac{B^{2}}{B_{H}^{2}} \bar{x}^{2}\right) q=0 .
$$

In Fig. 4(a), the normalized limiting current in a crossed-field gap as a function of $\lambda$ for $B / B_{H}=0,1$, and 2 at $\phi_{g}=1$ (solid lines). The dashed lines are the previous calculation without 


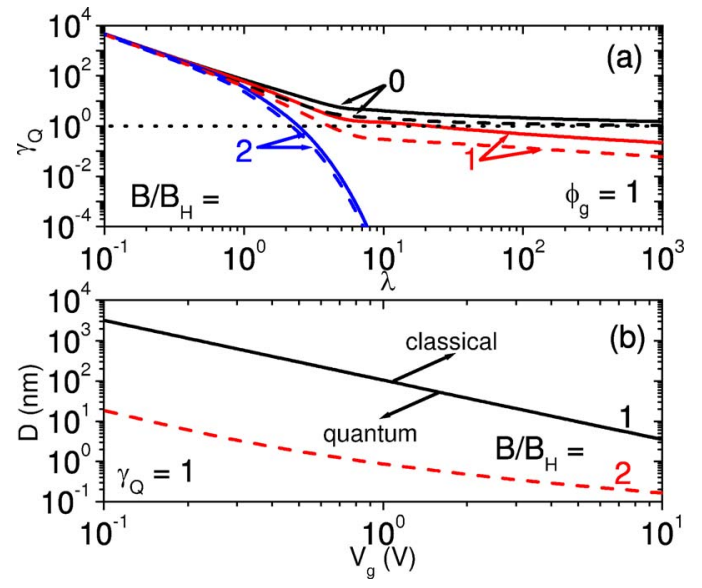

FIG. 4. (Color online) (a) The normalized unipolar SCL electron current density $\gamma_{Q}=J / J_{\mathrm{CL}}$ for $\phi_{g}=1$ (solid lines) and $\phi_{g} \gg 1$ (dashed lines) when $B / B_{H}=0,1$, and 2 ; (b) the boundary of the transition from quantum region (small $D$ ) to the classical region (large $D$ ) for $B / B_{H}=1$ and 2, as a function of $V_{g}=0.1$ to 10 volts.

the exchange-correlation effects, ${ }^{27}$ and the dotted line $\gamma_{Q}=1$ is the classical CL law. Thus, we see that $\gamma_{Q}$ remains finite for small $\lambda$, and it decreases with large $\lambda$. In Fig. 4(b), we show the boundary of the transition between the quantum regime and classical regime for magnetic insulation at $B / B_{H}=1$ and 2 , where the boundary is defined as the calculated values of $D$ at $\gamma_{Q}=1$ for a given gap voltage $V_{g}=0.1-10 \mathrm{~V}$.

\section{Surface properties}

In the previous quantum model of SCL flow, ${ }^{25}$ the electron emission mechanisms in the vicinity of the surface have been ignored, that is, the surface properties of the materials are not included. Note that the surface properties may become important when gap spacing is extremely small, such as $D<1 \mathrm{~nm}$. One of the possibilities to obtain SCL electron flow at the quantum regime is to use a field emitter with a low work function and/or with a sharp tip to emit high current electron beam at a low voltage within a nanoscale gap. From the SCL quantum models that have been presented (without including the surface properties), the enhancement of SCL current density increases with small gap voltage. However, the enhancement must have a threshold voltage since the emission mechanisms may not have the capability to reach the SCL condition at low voltage. By including the surface properties of metallic emitters, we will show some results to illustrate the threshold voltages to reach quantum SCL flow by considering a simple FN like electron field emission mechanism with the following assumptions. Note that detailed analysis for the transition from the FN emission to SCL flows in the quantum regime is beyond the scope of this paper, and will be the subject for further studies.

The assumptions are (a) the 1D quantum SCL model is valid to account for the space-charge effects of field emitted electrons; (b) all the electrons are emitted from the Fermi energy at zero temperature with a fixed work function $\phi_{\mathrm{WF}}$ independent of the applied electric field; (c) the geometrical enhancement $(\beta)$ of sharp emitter is accounted by an effec-

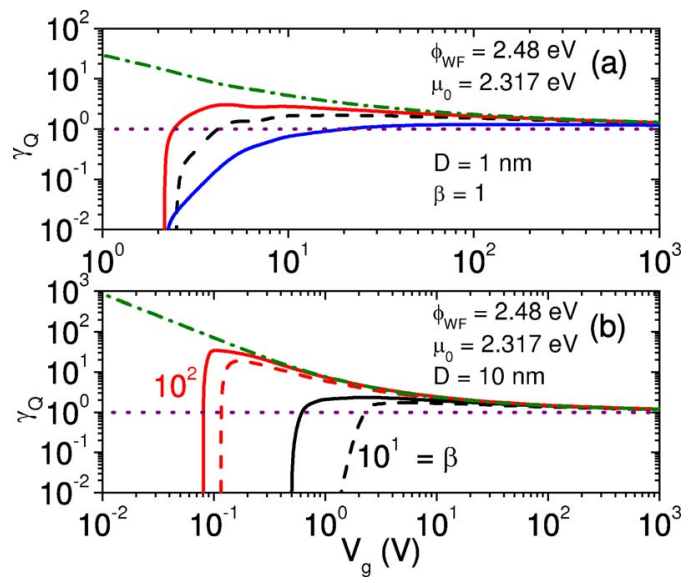

FIG. 5. (Color online) The normalized unipolar SCL electron current density $\gamma_{Q}=J / J_{\mathrm{CL}}$ as a function of $V_{g}$ for a Ba field emitter at various $D$ and $\beta$. The solid lines denote the calculations including image-charge potential with exchange-correlation (top) and without exchange-correlation (bottom) potential. The dashed lines denote the cases where only the exchangecorrelation potential is included (i.e., without image-charge potential). The dashed-dotted lines are quantum CL law with zero work function or at $\beta$ $\gg 1$. The dotted lines are the classical CL Law of $\gamma_{Q}=1$.

tive work function $\Phi_{\text {eff }}=\phi_{\mathrm{WF}} / \beta^{2 / 3}$; and (d) the normalized image charge potential near to the field emitter is $k /\left(\bar{x}+\bar{x}_{0}\right)$, where $k=e^{2} /\left(16 \pi \epsilon_{0} D\right)$ and

$$
\bar{x}_{0}=\frac{1}{D} \sqrt{\frac{\hbar^{2}}{2 m_{e}\left(\mu_{0}+\Phi_{\text {eff }}\right)}}
$$

represents a change in the metal-vacuum interface due to a global shift in the electron density as a result of finite barrier height, ${ }^{32}$ where $\mu_{0}$ is the chemical potential (or Fermi energy level at zero temperature). Under the assumptions, the surface properties such as the work-function and the imagecharge potential are included and Eq. (4) becomes

$$
q^{\prime \prime}+\lambda^{2}\left(\phi-\frac{V_{\mathrm{xc}}}{\phi_{g}}-\frac{4}{9} \frac{\gamma^{2}}{q^{4}}-\frac{\Phi_{\mathrm{eff}}}{\phi_{g}}+\frac{k}{\phi_{g}\left(\bar{x}+\bar{x}_{0}\right)}\right) q=0 .
$$

The boundary condition of $q(1)$ is modified to

$$
q(1)=\sqrt{2 \gamma / 3} /\left(1-\frac{\Phi_{\text {eff }}}{\phi_{g}}+\frac{k}{\phi_{g}\left(\bar{x}+\bar{x}_{0}\right)}\right)^{1 / 4} .
$$

The $\gamma_{Q}$ of the SCL electron flow with surface properties is determined for a given $V_{g}, D, \phi_{\mathrm{WF}}, \mu_{0}$, and $\beta$.

To illustrate the importance of surface properties at small $D$, the normalized electron current density $\gamma_{Q}$ is plotted as a function of $V_{g}$ in Figs. 5 for a barium (Ba) field emitter with a work function of $\phi_{\mathrm{WF}}=2.48 \mathrm{eV}$ and $\mu_{0}=2.317 \mathrm{eV}$ at (a) $D=1 \mathrm{~nm}$ and $\beta=1$, and (b) $D=10 \mathrm{~nm}$ and $\beta=10$ and 100 . The solid lines denotes the calculations including imagecharge potential with exchange-correlation (top) and without exchange-correlation (bottom) potential. The dashed lines denote the case where only the exchange-correlation potential is included (i.e., without image-charge potential). The dashed-dotted and dotted lines are, respectively, the quantum CL law without including the surface properties, and the classical CL law of $\gamma_{Q}=1$. From the figure, the $\gamma_{Q}$ increases sharply when $V_{g}$ is higher than a threshold value, and it 


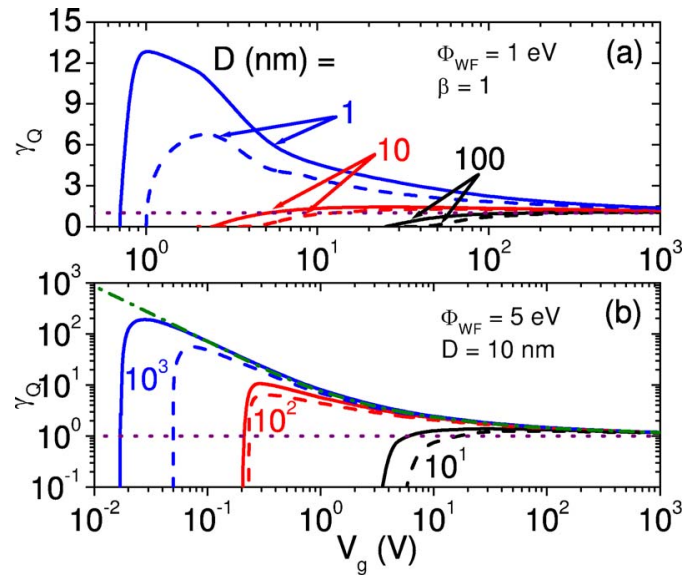

FIG. 6. (Color online) The normalized unipolar SCL electron current density $\gamma_{Q}=J / J_{\mathrm{CL}}$ as a function of $V_{g}$ for an field emitter with (a) a low work function $\phi_{\mathrm{WF}}=1 \mathrm{eV}$, and (b) a high work function $\phi_{\mathrm{WF}}=5 \mathrm{eV}$, at various $D$ and $\beta$. The solid and dashed lines are with and without image-charge potential. The dashed-dotted lines are quantum CL law with zero work function or at $\beta \gg 1$. The dotted lines are the classical CL Law of $\gamma_{Q}=1$.

saturates at $\gamma_{Q} \approx 1$ for high $V_{g}$. If the field emission current is sufficiently high, $\gamma_{Q}$ may reach the quantum CL law, which is larger than the classical limit of $\gamma_{Q}=1$ (dotted line). At $D=1 \mathrm{~nm}$ and $\beta=1$, the maximum value is $\gamma_{Q} \approx 3$ at $V_{g}$ $\approx 4 \mathrm{~V}$ with an effective electrical field $=\beta \times V_{g} / D$ $=4 \mathrm{~V} / \mathrm{nm}$. At $D=10 \mathrm{~nm}$ and $\beta=100$, we have $\gamma_{Q} \approx 30$ at $V_{g} \approx 0.1 \mathrm{~V}$ with the same order of $\beta \times V_{g} / D=1 \mathrm{~V} / \mathrm{nm}$.

Sufficiently large quantum SCL current density may also be reached with a flat emitter of low work function or a sharp emitter of high work function. In Fig. 6, we show the calculations for two cases: (a) A low work function emitter $\left(\phi_{\mathrm{WF}}=1 \mathrm{eV}\right)$ at $\beta=1$ for $D=1,10$, and $100 \mathrm{~nm}$, and (b) a high work function emitter $\left(\phi_{\mathrm{WF}}=5 \mathrm{eV}\right)$ at $D=10 \mathrm{~nm}$ for $\beta=10,100$, and 1000. Thus, high current field emission in a nano diode may have a limiting current value that is larger than the classical SCL values $\gamma_{Q}=1$, which have been calculated by using the classical theory. ${ }^{33}$

\section{CONCLUSION}

In conclusion, we have formulated a new quantum model for the space-charge-limited (SCL) electron flow in a planar 1D nanogap with small gap voltage. It is found that the classical concepts of SCL electron flow, such as limiting current density, bipolar flow, transit time, beam-loaded capacitance, magnetic insulation and surface properties are no longer valid in the quantum regime, where quantum effects become important. A new quantum scaling of $V_{g}^{1 / 2}$ and $D^{-4}$ has been predicted for low $V_{g}$ and small $D$. New findings of beam-loaded capacitance, minimal electron transit time in quantum regime, and the revised quantum CL law including surface properties of various field emitters are also presented. A smooth transition between the classical and the quantum regimes has been demonstrated.

Note that the Heisenberg uncertainty principle is important in determining the properties of the SCL flow in quan- tum regime, such as momentum and energy of the transmitted SCL electrons at anode $(x=D)$ with a gain of kinetic energy $E=e V_{g}$. Assuming that the accuracy in determining the position and time (of the electrons) are $\Delta x=D$ and $\Delta t=T$ (transit time), the accuracy in measuring the momentum and energy are $\Delta p / p \geqslant 1 /(2 \lambda)$ and $\Delta E / E \geqslant \tau^{-1} /(2 \lambda)$, where $\lambda$ is the ratio of the gap spacing to the electron de Broglie wavelength, and $\tau$ is the normalized transit time [see Eq. (9)]. As an example at $D=1 \mathrm{~nm}, V_{g}=1 \mathrm{~V}$, we have $\tau=0.523$ [from Fig. 3(c)], and the uncertainties are $\Delta p / p$ $\geqslant 0.1$ and $\Delta E / E \geqslant 0.19$.

\section{ACKNOWLEDGMENTS}

This work was partly supported by the Agency for Science, Technology and Research of Singapore (Reference number 042101 0080). One of the authors (L. K. A.) would like to acknowledge the support of Los Alamos National Laboratory operated by the University of California for USDOE when he was a visiting scientist during the course of this work.

${ }^{1}$ C. D. Child, Phys. Rev. 32, 492 (1911).

${ }^{2}$ I. Langmuir, Phys. Rev. 2, 450 (1913).

${ }^{3}$ I. Langmuir, Phys. Rev. 23, 347 (1923).

${ }^{4}$ G. Jaffe, Phys. Rev. 44, 91 (1944).

${ }^{5}$ I. Langmuir, Phys. Rev. 33, 954 (1929).

${ }^{6}$ H. R. Jory and A. W. Travelpiece, J. Appl. Phys. 40, 3924 (1969).

${ }^{7}$ Y. Y. Lau, D. Chernin, D. G. Colombant, and P.-T. Ho, Phys. Rev. Lett. 66, 1446 (1991).

${ }^{8}$ A. Valfells, D. W. Feldman, M. Virgo, P. G. O’Shea, and Y. Y. Lau, Phys. Plasmas 9, 2377 (2002).

${ }^{9}$ P. V. Akimov and H. Schamei, J. Appl. Phys. 92, 1690 (2002).

${ }^{10}$ M. D. Nijkerk and P. Kruit, J. Appl. Phys. 96, 2985 (2004).

${ }^{11}$ R. R. Puri, D. Biswas, and R. Kumar, Phys. Plasmas 11, 1178 (2004).

${ }^{12}$ D. Biswas, R. Kumar, and R. R. Puri, Phys. Plasmas 12, 093102 (2005).

${ }^{13}$ R. J. Umstattd, C. G. Carr, C. L. Frenzen, J. W. Luginsland, and Y. Y. Lau, Am. J. Phys. 73, 160 (2005).

${ }^{14}$ J. W. Luginsland, Y. Y. Lau, and R. M. Gilgenbach, Phys. Rev. Lett. 77, 4668 (1996).

${ }^{15}$ Y. Y. Lau, Phys. Rev. Lett. 87, 278301 (2001).

${ }^{16}$ R. J. Umstattd and J. W. Luginsland, Phys. Rev. Lett. 87, 145002 (2001).

${ }^{17}$ J. J. Watrous, J. W. Luginsland, and M. H. Frese, Phys. Plasmas 8, 4202 (2001).

${ }^{18}$ K. G. Kostov and J. J. Barroso, Phys. Plasmas 9, 1039 (2002).

${ }^{19}$ A. Rokhlenko and J. L. Lebowitz, Phys. Rev. Lett. 91, 085002 (2003).

${ }^{20}$ J. W. Luginsland, Y. Y. Lau, R. J. Umstatt, and J. J. Watrous, Phys. Plasmas 9, 2371 (2002).

${ }^{21}$ W. S. Koh, L. K. Ang, and T. J. T. Kwan, Phys. Plasmas 12, 053107 (2005).

${ }^{22}$ H.-L. Lee, S.-S. Park, D.-I. Park, S.-H. Hahm, and J.-H. Lee, J. Vac. Sci. Technol. B 16, 762 (1998).

${ }^{23}$ A. A. G. Driskill-Smith, D. G. Hasko, and H. Ahmed, Appl. Phys. Lett. 75, 2845 (1999).

${ }^{24}$ P. Steinmann and J. M. R. W. k, Appl. Phys. Lett. 86, 063104 (2005).

${ }^{25}$ L. K. Ang, T. J. T. Kwan, and Y. Y. Lau, Phys. Rev. Lett. 91, 208303 (2003).

${ }^{26}$ L. K. Ang, T. J. T. Kwan, and Y. Y. Lau, IEEE Trans. Plasma Sci. 32, 410 (2004).

${ }^{27}$ L. K. Ang, T. J. T. Kwan, and Y. Y. Lau, Phys. Rev. E 64, 017501 (2001).

${ }^{28}$ J. P. Perdew and Y. Wang, Phys. Rev. B 45, 13244 (1992).

${ }^{29}$ W. S. Koh, L. K. Ang, S. P. Lau, and T. J. T. Kwan, Appl. Phys. Lett. 87, 193112 (2005).

${ }^{30}$ C. Bracher, M. Kleber, and M. Riza, Phys. Rev. A 60, 1864 (1999).

${ }^{31}$ A. W. Hull, Phys. Rev. 18, 31 (1921).

${ }^{32}$ K. L. Jensen, J. Appl. Phys. 85, 2667 (1999).

${ }^{33}$ Y. Y. Lau, Y. F. Liu, and R. K. Parker, Phys. Plasmas 1, 2082 (1994). 